\title{
Effect of Lycopene Supplementation on Oxidative Stress: An Exploratory Systematic Review and Meta-Analysis of Randomized Controlled Trials
}

\author{
Jinyao Chen, Yang Song, and Lishi Zhang \\ Department of Nutrition and Food Hygiene, West China School of Public Health, Sichuan University, Chengdu, China.
}

\begin{abstract}
Lycopene is a potentially useful compound for preventing and treating cardiovascular diseases and cancers. Studies on the effects of lycopene on oxidative stress offer insights into its mechanism of action and provide evidence-based rationale for its supplementation. In this analysis, randomized controlled trials of the effects of oral lycopene supplementation on any valid outcomes of oxidative stress were identified and pooled through a search of international journal databases and reference lists of relevant publications. Two reviewers extracted data from each of the identified studies. Only studies of sufficient quality were included. Twelve parallel trials and one crossover trial were included in the systematic review, and six trials provided data for quantitative meta-analysis. Our results indicate that lycopene supplementation significantly decreases the DNA tail length, as determined using comet assays, with a mean difference (MD) of -6.27 [95\% confidence interval (CI) $-10.74,-1.90](P=.006)$ between the lycopene intervention groups and the control groups. Lycopene supplementation does not significantly prolong the lag time of low-density lipoprotein (MD 3.76 [95\% CI -2.48, 10.01]; $P=.24$ ). Lycopene possibly alleviates oxidative stress; however, biomarker research for oxidative stress needs be more consistent with the outcomes in lycopene intervention trials for disease prevention.
\end{abstract}

KEY WORDS: • lycopene $\bullet$ meta-analysis $\bullet$ oxidative stress $\bullet$ randomized controlled trial $\bullet$ systematic review

\section{INTRODUCTION}

C ARDIOVASCUlar DISEASES AND CANCERS are main contributors to human morbidity and mortality, and the pivotal role of oxidative stress in the etiology of both diseases is well known. Oxidative stress is defined as disturbances in the prooxidant-antioxidant balance that favors oxidation. Thus, oxidative stress is essentially an imbalance between the production of various reactive species and the ability of the natural protective mechanisms of organisms to cope with these reactive compounds and to prevent adverse effects. ${ }^{1}$ Oxidative status is assessed in terms of the overall oxidative/reductive potency of a given specimen (e.g., blood or urine) or the susceptibility of various oxidizable components to ex vivo peroxidation. ${ }^{1-3}$

Lycopene is a potentially powerful antioxidant because of its conjugated double bonds. In vitro evidence suggests that lycopene protects lipoproteins and vascular cells from oxidation, but in vivo evidence is limited. ${ }^{2,4}$ Lycopene reportedly has the strongest singlet oxygen-quenching capacity among carotenoids, ${ }^{5}$ and is the most effective quencher in

Manuscript received 16 November 2012. Revision accepted 28 February 2013.

Address correspondence to: Lishi Zhang, MD, West China School of Public Health, Sichuan University, No. 16, third section, S. Renmin Rd., Chengdu 610041, Sichuan, China, E-mail: lishizhang_56@163.com plasma, low-density lipoprotein (LDL), and human lymphoid cells in vitro. ${ }^{4,6}$ Epidemiologic studies have reported that the consumption of lycopene-rich foods decreases the risk of cardiovascular diseases and cancers. ${ }^{7-9}$ Several clinical trials have provided evidence that lycopene lowers oxidative stress, particularly by preventing LDL oxidation. ${ }^{4,10,11}$ However, in 2000, the panel on Dietary Reference Intake evaluated the potential health effects of $\beta$-carotene and other carotenoids and concluded that no convincing evidence indicates that substantially increasing the carotenoid intake above current levels significantly affects the antioxidant status; however, lycopene was not specifically addressed. ${ }^{12}$ Considering that lycopene metabolism has not been fully elucidated and antioxidative nutrients interact with each other during gastrointestinal absorption and metabolism, the function of lycopene in vivo possibly differs from that in vitro. ${ }^{13-16}$

Decades of clinical and preclinical studies have broadened our understanding of lycopene and its use in numerous chronic, oxidative stress-induced pathologies; however, the results of these studies have shown mixed results, although with promising implications on the efficacy of lycopene in vivo. ${ }^{13,17,18}$ To provide more convincing estimates, this systematic review and meta-analysis assesses the effect of lycopene supplementation on oxidative stress. A wide variety of functional assays for oxidative stress is covered, 
including various measures of lipid oxidation, DNA oxidation, and protein oxidation, for illustrative purposes. This article is the first systematic review on this topic.

\section{METHODS AND MATERIALS}

\section{Search strategy}

OVID MEDLINE, EMBASE, Web of Science, Springer, CNKI, and CENTRAL (The Cochrane Library) were systematically searched for relevant studies published until June 2012. Further searches were done by scanning the reference lists of original studies, meta-analyses, and review publications on lycopene and tomato. No language or other limitations were imposed. Figure 1 illustrates the selection process. Terms used for MEDLINE (and adopted for other databases) in both subject heading and body text were:

1. Lycopene, carotenoids, antioxidants, tomato, or dietary

2. LDL oxidation, LDL peroxidation, DNA damage, or oxidative stress

3. Both (1) and (2)

4. Limit (3) to humans

Studies were included if they met the following criteria: (1) randomized controlled trials (RCTs) on human subjects; (2) biomarkers of oxidative stress as outcomes were reported; (3) intervention included oral lycopene supplementation compared with a concurrent controlled group of placebo or no intervention treatments that were initiated at any time during the subject's lifetime; (4) the trials lasted at least 7 days. To reduce heterogeneity across interventions, trials of foods enriched with lycopene and/or unknown lycopene doses were excluded from this review. Supple-

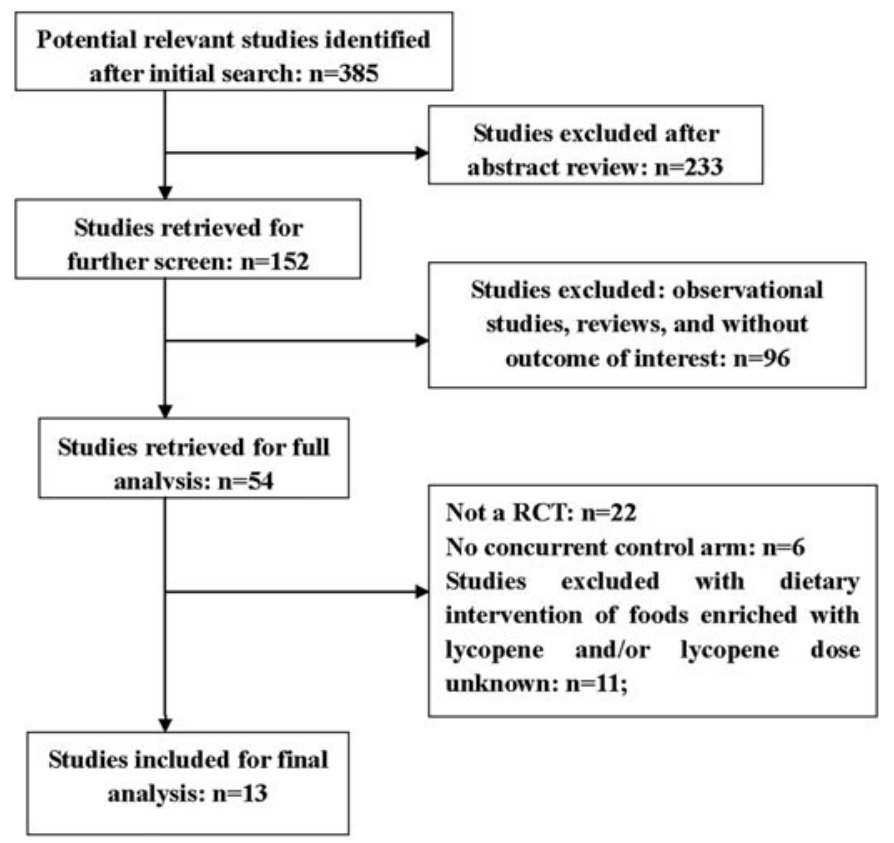

FIG. 1. Search strategy flow chart. mentation with purified lycopene or formulation with small quantities of other carotenoids and/or flavonoids was included because of their usual inclusion in lycopene supplements. Further, each of the included trials was required to state explicitly the word "random" in the description of treatment assignment. Further details regarding randomization methods used (blocking, random-number generation, etc.) were not required. Missing data were dealt with by contacting the investigators of the original study to request the missing data, or to provide further clarification on data. Analysis was performed on the available data in cases where the missing data were irretrievable. The selection of these biomarkers was based on previous research and reviews, which specified that DNA damage, antioxidant capacity of plasma, lipid peroxidation, protein oxidation, and antioxidative enzymes could be used as biomarkers for evaluating oxidative stress in vivo. ${ }^{19,20}$ Various functional in vivo and ex vivo assays were covered for illustrative purposes. The assays were further categorized into:

- LDL oxidation: LDL lag-time, LDL oxidation rate

- Lipid and protein peroxidation products: plasma malondialdehyde (MDA), thiobarbituric acid-reactive substances (TBARS), urinary 8-iso-PGF ${ }_{2 \alpha}$, plasma thiols.

- Plasma antioxidative capability: total antioxidant capability (TAC), ferric-reducing ability of plasma (FRAP), oxygen radical absorbance capacity (ORAC), P/S fatty acid ratio.

- Antioxidative enzymes: superoxide dismutase (SOD), catalase (CAT), glutathione peroxidase (GPx).

- DNA damage, DNA products (comet assay results, 8-OHdG, etc.).

\section{Data extraction}

The following information was retrieved from each included study:

- Characteristics of study participants

- Alterations in plasma lycopene

- Outcome measurements

- Trial design and blinding methods

- Intervention measures, type of control, and trial duration

- Allocation of concealment, description of randomization, evaluation of losses to follow-up, and subject compliance

- Dietary advice before and during treatment

The inclusion and exclusion criteria for the study participants were also extracted (data not presented).

\section{Quality assessment}

All trials were objectively assessed for the risk of bias by two independent reviewers (J.C. and Y.S.) as recommended by the Cochrane guidelines. ${ }^{21}$ The Cochrane protocol explicitly discourages the use of scales to assess the quality of trials when empirical support was lacking; thus, the quality of each trial included in this review was assessed using seven criteria. ${ }^{22}$ 


\section{Statistical analysis}

Data were extracted as baseline and endpoint means, standard deviations, and sample sizes for both intervention and placebo groups for each oxidative outcome. When testing was performed at multiple time points, only data from baseline and the final time point were extracted. For each trial following a parallel design, the effect was calculated as the difference in the baseline and end-trial levels between the intervention and the control groups; for crossover design, the effect was assessed using the data from the first period. ${ }^{23,24}$ A fixed-effect model was used to combine data where the studies are assumed to estimate the same underlying treatment effect. If clinical heterogeneity was sufficient to expect that the underlying treatment effects differed between trials, or if substantial statistical heterogeneity was detected, a random-effect model was adopted to produce an overall summary. The results of the metaanalysis are presented as forest plots.

Heterogeneity was assessed using both $\chi^{2}$ and $I^{2}$. The $\chi^{2}$ test assesses whether the differences in the results are due to chance only. The data were considered heterogeneous when the $P$-value is low. $I^{2}$ assesses the percentage of variability in the estimated effect that is due to heterogeneity rather than chance. $I^{2}$ values higher than $50 \%$ indicate substantial heterogeneity. ${ }^{21}$ Sensitivity analysis was performed to verify the results of the meta-analysis. Publication bias was assessed by inspecting funnel plots. The analysis was performed using Review Manager 5.0 software. ${ }^{25}$ Data reported in formats that did not allow entry into RevMan software were reported in tables or in text.

To investigate the differences in the results across studies, several prior hypotheses were developed as follows:

- Study population: normal or in a pathologic state, wherein larger treatment effects were found in the latter.

- Different methodological qualities: randomization concealed or unconcealed; blinded or unblinded; extent of losses to follow-up; dietary advice (whether taking lycopene-containing food or not; anticipating that effect size was overestimated by refraining from lycopene-containing food).

- Study duration: anticipating longer treatments induce stronger effects.

- Lycopene formulations and doses: hypothesizing that the magnitude of the effect varies with the formulation.

- Baseline plasma lycopene level: hypothesizing that the effect is more pronounced with lower baseline plasma lycopene levels.

\section{RESULTS}

\section{Description of included studies}

The characteristics of the 13 clinical trials ${ }^{26-38}$ meeting inclusion criteria are summarized in Table 1 . We excluded 22 trials for not having an RCT design, ${ }^{39-60} 6$ trials for the absence of concurrent controlled arms, ${ }^{61-66} 11$ trials for dietary intervention with food and/or no lycopene dose mentioned, ${ }^{67-77}$ and 2 trials for single-dose administration. ${ }^{78,79}$
These 13 trials were conducted between 1998 and 2011, comprising 844 participants. The trials were conducted in the United States, Canada, Ireland, Spain, Germany, Italy, Iran, France, and Korea. The trial size ranged from 26 to 175 participants (mean age of 25.9-70 years; mean body-mass index [BMI] of $21.0-28.5 \mathrm{~kg} / \mathrm{m}^{2}$ across 10 trials). In terms of trial design, one of the 13 trials was designed as a crossover: ${ }^{32}$ placebo/washout/lycopene and lycopene/washout/ placebo for two groups, with an intermittent washout period of 26 days. The remaining 12 trials were designed as parallel trials, ${ }^{26-31,33-38}$ particularly the trial by Talvas et al. ${ }^{36}$ which was designed as a two-phase RCT: red tomato extract versus yellow tomato extract as the first phase and lycopene capsule versus placebo as the second phase, with a washout period of 2 weeks. The second phase was included in this review as a parallel design. Among the included studies, eight trials were designed as double-blind, ${ }^{28,31-37}$ two trials were singleblind, ${ }^{29,36}$ and three trials reported no information regarding blinding, ${ }^{26,27,30}$ as described in Tables 1 and 2 .

Six trials administered one lycopene dosage as the only treatment arm, ${ }^{30-32,34,36,38}$ three trials designed different lycopene doses as several treatment arms, ${ }^{26,35,37}$ whereas four trials designed treatment arms with other carotenoid supplements. ${ }^{27-29,33}$ The lycopene formulations differed across the studies, as shown in Table 1, which could be categorized into the following four formulations: (1) purified lycopene; ${ }^{33,34,36}$ (2) lycopene with small quantities of tocopherol; ${ }^{28,35}$ (3) lycopene capsules containing $10 \% \beta$-carotene, ${ }^{27,29}$ and (4) lycopene with its metabolites (phytoene and phytofluene) and small quantities of tocopherol and $\beta$-carotene. ${ }^{26,30-32,37,38}$ Trial participants were heterogeneous, varying from healthy adults, ${ }^{26-29,31-33,36-38}$ the most common subjects, to other particular populations such as those with newly diagnosed prostate cancer, ${ }^{30}$ with type 2 diabetes mellitus, ${ }^{34}$ or with mildly elevated cholesterol levels. ${ }^{35}$

For missing data, in the trial by Mackinnon et al., ${ }^{26}$ the three lycopene treatment arms were merged into one LYCOPENE group in the original article. We used the merged data in this systematic review, because the original data for each arm were unavailable. The formulation of lycopene supplementation in the trial of Neyestani et al. ${ }^{34}$ was irretrievable, whereas the review by Erdman et al. ${ }^{13}$ identified the lycopene source in the same article as purified lycopene. In the present study, we designated the lycopene source as purified lycopene.

\section{Quality assessment}

The quality of the trials was assessed, as shown in Table 2. The majority of trials did not report details regarding concealment allocation (12 trials) and randomization method (12 trials). Only one trial ${ }^{38}$ reported an appropriate blinding method. Except for the trial by Carroll et al., ${ }^{29}$ the other 12 trials mentioned the methods for evaluating participant compliance with intervention protocol, and seven trials discussed losses to follow-up. ${ }^{26,29,31,35-38}$ With regard to dietary advice, 11 trials described a specific instruction; ${ }^{26,27,30-38}$ among them, three trials instructed subjects to 


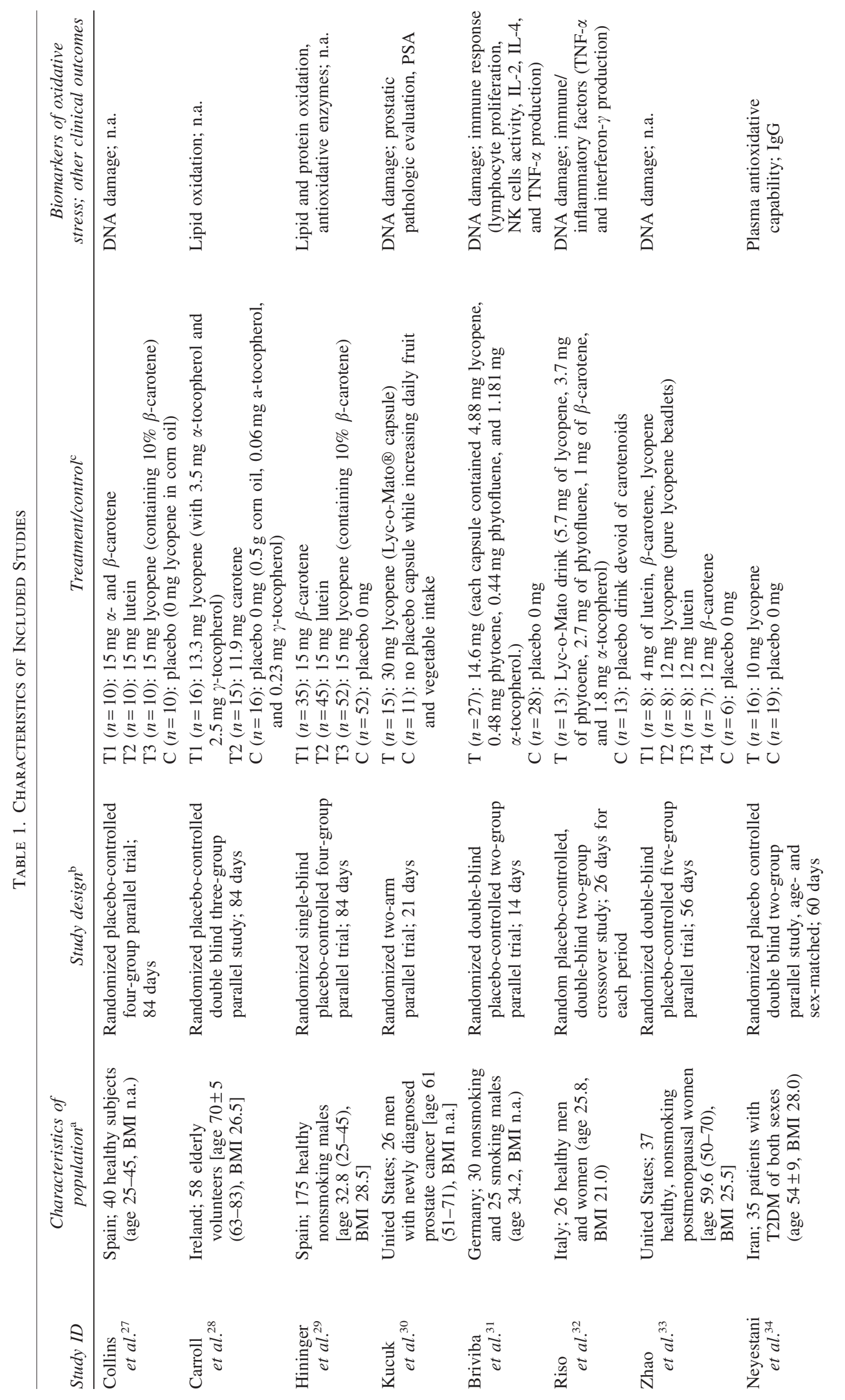




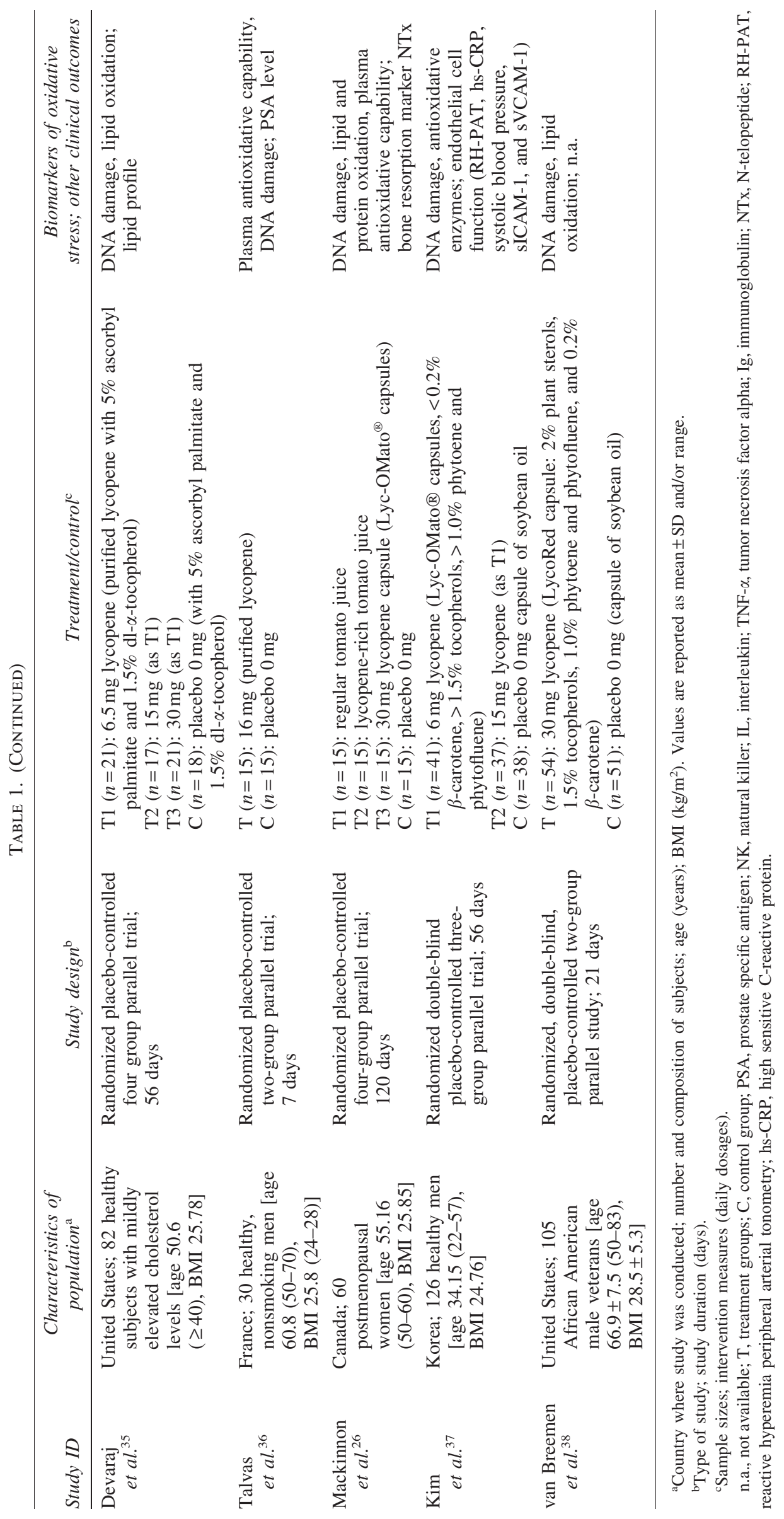




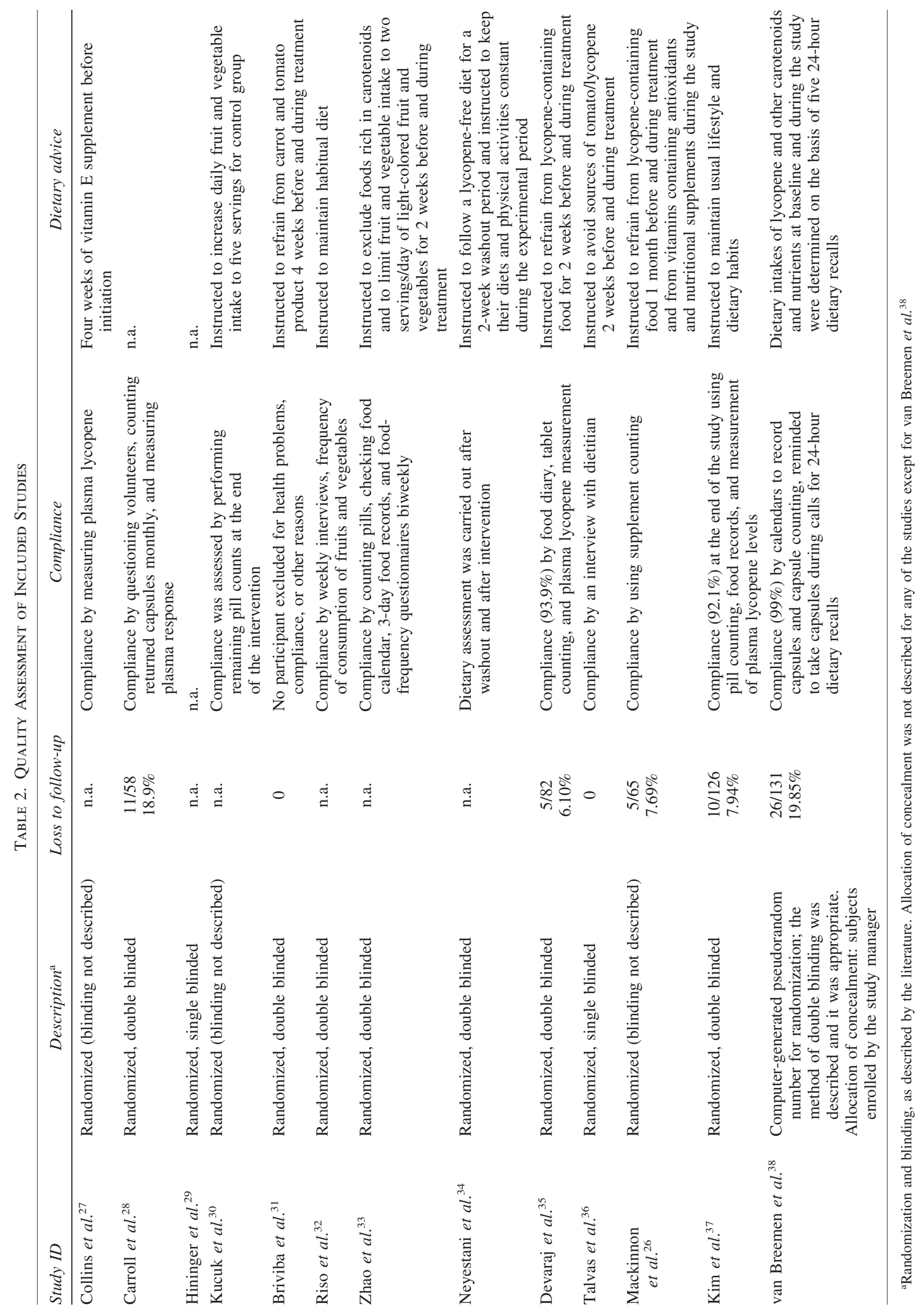


avoid lycopene-containing food during the washout and treatment periods, ${ }^{26,35,36}$ one trial instructed subjects to refrain from lycopene-containing food during the washout period and to keep a constant diet during treatment period $;^{34}$ two trials asked subjects to refrain from food enriched with lycopene and carotenoids; ${ }^{31,33}$ two trials encouraged subjects to maintain their diets and lifestyle, ${ }^{32,37}$ which possibly inflated the effect size; one trial provided vitamin E supplement before initiation, ${ }^{27}$ one trial instructed the controlled subjects to increase fruit and vegetable intake, possibly resulting in underestimation of the effect size, ${ }^{30}$ and one trial instructed the subjects to keep records of lycopene and carotenoid intake. ${ }^{38}$ Compliance and dietary monitoring were satisfactory in all evaluated trials.

\section{Plasma/tissue lycopene levels}

Except for one trial, ${ }^{32}$ the other 12 studies reported alterations in plasma lycopene levels, among which seven trials provided explicit data on pretreatment and posttreatment. ${ }^{26,28-31,36,38}$ Plasma lycopene at baseline ranged from 0.053 to $0.69 \mu \mathrm{M}$, as shown in Table 3. Among the 12 studies, two reported prostate lycopene levels ${ }^{30,38}$ and one reported the LDL and high-density lipoprotein concentrations of lycopene. ${ }^{28}$

Plasma and tissue lycopene in the lycopene intervention groups of all trials was significantly elevated after supplementation, which further supported the validity of these clinical trials (Table 3).

\section{Oxidative biomarker assessment}

The oxidative parameters assessed in each study were categorized and are presented in Table 4. In total, at least 20 oxidative parameters were applied across these studies, as summarized below.

LDL oxidation. Three trials assessed the LDL lag time, ${ }^{28,29,35}$ and one reported the LDL oxidation rate. ${ }^{35}$ These data were considered appropriate for meta-analysis (Table 5). With a total pooled sample size of 167 subjects, the lycopene treatment did not significantly prolong the LDL lag time (MD 3.76 [95\% confidence interval (CI) $-2.48,10.01] ; P=.24)$. A considerable heterogeneity was observed $\left(I^{2}=100 \% ; P<.00001\right)$.

Lipid and protein oxidation and peroxidation products. Lipid oxidation products were investigated in five trials ${ }^{26,32,35,36,38}$ with the same test principle for plasma MDA, hydroxyl nonenal (HNE), and TBARS, and two trials for urinary 8 -iso- $\mathrm{PGF}_{2 \alpha .}{ }^{32,36}$ Except for one trial, ${ }^{26}$ which reported increased TBARS versus baseline in the LYCOPENE group, no other significant effect of lycopene on lipid peroxidation products compared with the placebo or baseline level was reported. For protein oxidation products, two trials reported thiol groups in different measurement units, as $\mu \mathrm{mol} / \mathrm{g}$ proteins in one triall ${ }^{29}$ and $\mu \mathrm{M}$ in plasma in another. ${ }^{26} \mathrm{~A}$ significantly positive effect was found between the post-treatment level with the baseline level of the LYCOPENE group in the latter trial.
Antioxidative enzymes and plasma antioxidative capability. Antioxidative enzymes were investigated in three trials. ${ }^{26,29,37}$ No significant effect was reported in GPx, glutathione disulfide (GSSG), glutathione (GSH), and SOD, except that one trial ${ }^{37}$ reported increased SOD versus baseline in an intervention arm of $15 \mathrm{mg} /$ day lycopene $(P<.05)$.

FRAP, ${ }^{36}$ ORAC, ${ }^{36} \mathrm{TAC}^{26,34}$ and $\mathrm{P} / \mathrm{S}$ fatty acid ratio ${ }^{29}$ were adopted to evaluate plasma antioxidative capability. One trial $^{26}$ reported increased TAC versus baseline in the LYCOPENE group. However, no significant effect was found in other parameters.

DNA damage (by Comet assay or products). Three trials $^{32,33,35}$ assessed the lymphocyte DNA tail length, and the data were considered appropriate for meta-analysis (Table 5). With a pooled sample size of 115 subjects, lycopene was found to decrease the DNA tail length significantly, which was measured using a comet assay (MD -6.27 [95\% CI $-10.74,-1.90] ; P=.006)$. Considerable heterogeneity $\left(I^{2}=100 \% ; P<.00001\right)$ was observed across the three studies. The urinary 8 -OHdG, ${ }^{35}$ plasma 8 -OHdG, ${ }^{27}$ and 8-oxo-dG/ $10^{6} \mathrm{dG}$ levels in prostate tissue,,$^{38}$ as well as peripheral blood lymphocyte level of 5-OHmdU ${ }^{30}$ were determined in four trials. Except for Devaraj et al., ${ }^{35}$ who found decreased urinary $8-\mathrm{OHdG}$ versus baseline at $30 \mathrm{mg} / \mathrm{d}$ lycopene, no other significant effect was reported in the other four trials.

\section{Clinical outcomes}

The clinical outcomes assessed in these trials were also analyzed, and they are listed in Table 1. Clinical outcomes were evaluated in eight trials:

- Lipid profile: No significant differences were found. ${ }^{35}$

- Prostate cancer-related pathologic status: In the trial by Kucuk et al.,$^{30}$ the pathologic stage of prostate cancer in the lycopene intervention group was less advanced and had lower PSA. In the trial by Talvas et al. ${ }^{36}$ the PSA levels in the healthy men did not change after lycopene intervention.

- Inflammatory/immune factors: IL-4 production was reduced by lycopene intervention, ${ }^{31} \mathrm{TNF}-\alpha$ production was reduced by lycopene intervention, ${ }^{32}$ and $\operatorname{IgG}$ increased after lycopene intervention. ${ }^{34}$

- Bone resorption marker (NTx): NTx was reduced after lycopene intervention. ${ }^{26}$

- Endothelial cell function: An increase in reactive hyperemia peripheral arterial tonometry (RH-PAT) index from baseline, whereas high-sensitive C-reactive protein (hs-CRP), systolic blood pressure, soluble intercellular adhesion molecule-1 (sICAM-1), and soluble vascular cell adhesion molecule-1 (sVCAM-1) were significantly decreased in the $15 \mathrm{mg} / \mathrm{d}$ group. ${ }^{37}$

\section{Sensitivity analysis and subgroup analysis}

Sensitivity analysis was performed by excluding certain studies to examine the influence of the omitted study on the pooled effect. Thus, the overall effects were 1.15 ( -7.37 to 


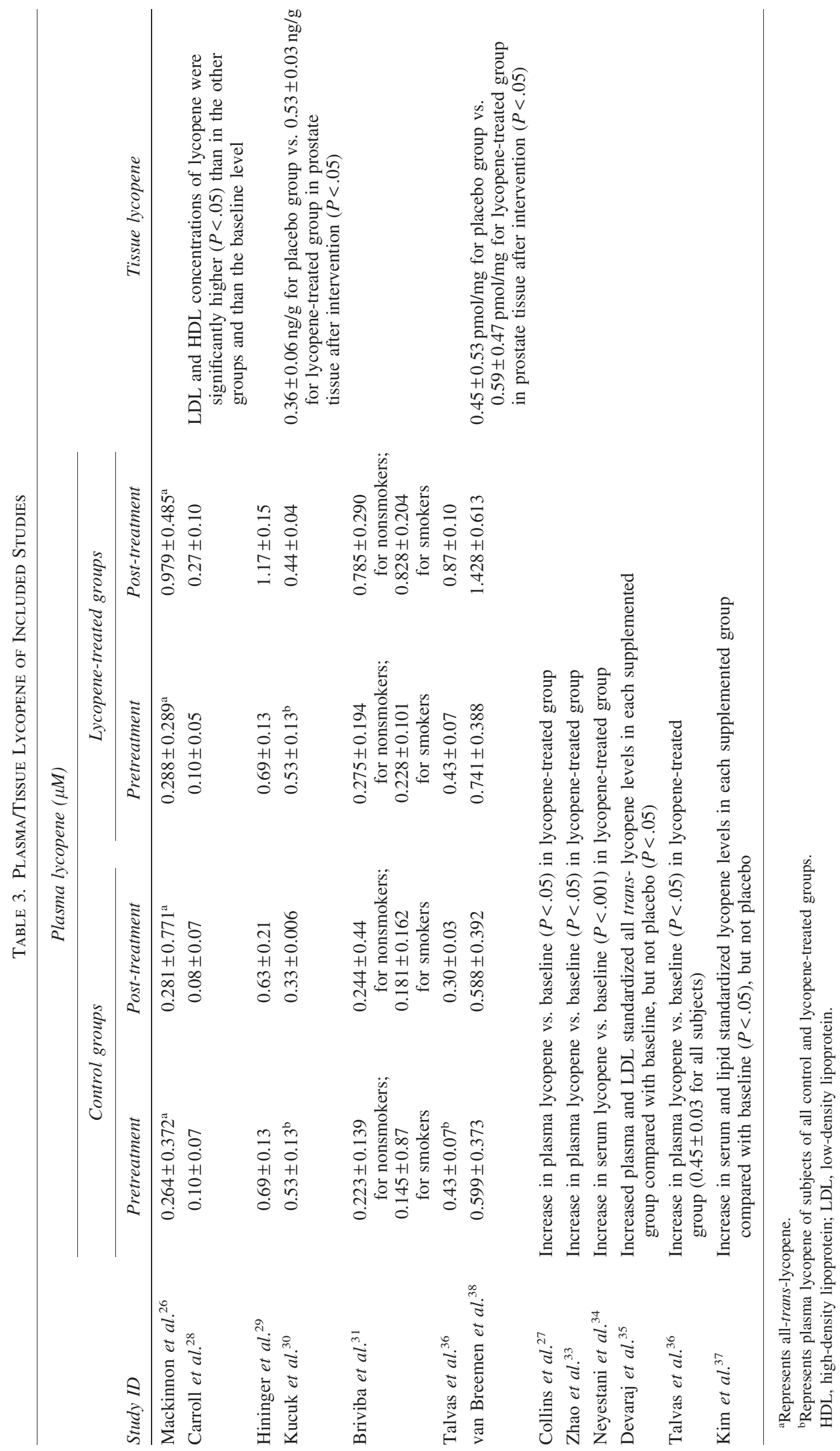


Table 4. Categorized Oxidative Parameters of Each Study

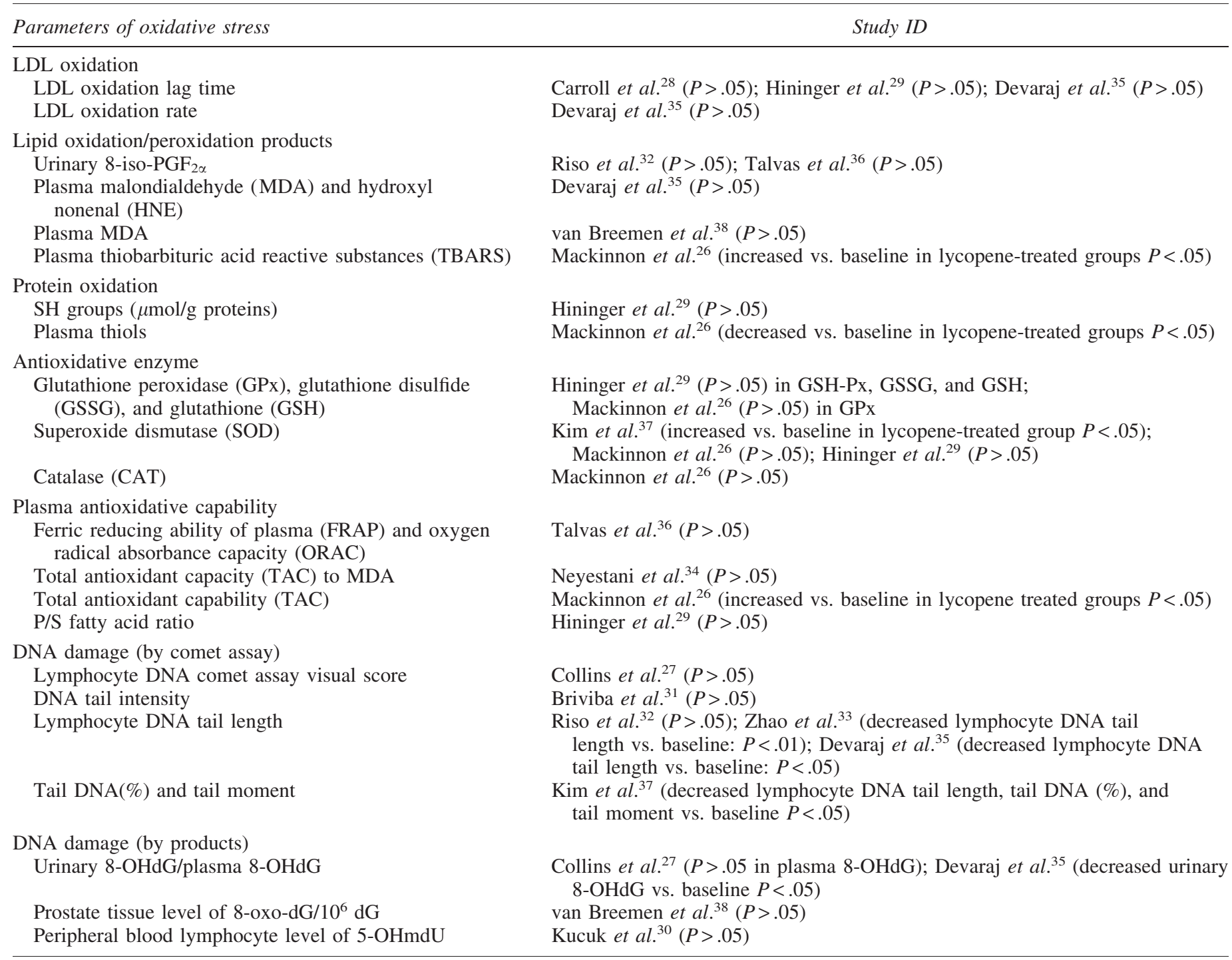

$P>.05$ means no significant difference with placebo groups or baseline.

9.68), 2.90 ( -9.06 to 14.85 ), and 7.24 (3.81 to 10.27 ), respectively, with the exclusion of trials 28,29 , and 35 on the extension of the LDL lag time. With exclusion of trials 37,32 , and 33, the effects on the DNA tail length were -3.55 $(-8.94$ to 1.84$),-9.05$ ( -14.53 to -3.57$)$, and -6.32 ( -17.19 to 4.55$)$, respectively. No quantitative subgroup analysis was conducted because of the small number of included studies on the same parameter. Funnel plots were not constructed because of the small number of included studies.

\section{DISCUSSION}

\section{Summary of main results}

The present study is the first systematic review of the antioxidative effects of lycopene, in which all available RCTs on the subject were pooled. This review included 13 RCTs, which vary in participant characteristics, formula- tions, and intervention measures of lycopene, parameters of oxidative stress, and study duration. Pooling the results of the three trials revealed no statistically significant difference in the LDL lag time between the lycopene treatment group and the controlled group. However, a meta-analysis of another three trials suggested that lycopene significantly decreases the DNA tail length as measured by comet assays. Other meta-analyses were impossible to carry out, because the contributing data for other outcomes came from only one trial. Comparisons of the effect of purified lycopene with other formulations and dosage/duration of lycopene is statistically impossible. However, the results showed no obvious discrepancy based on this systematic review.

Oxidative DNA damage is a potential marker for monitoring oxidative stress, as well a marker of oxidative disease risk, $,, 19,20$ one of the major and well-characterized functions of lycopene antioxidative activity. Despite the uncertainty posed by the limited population size and the heterogeneity in 


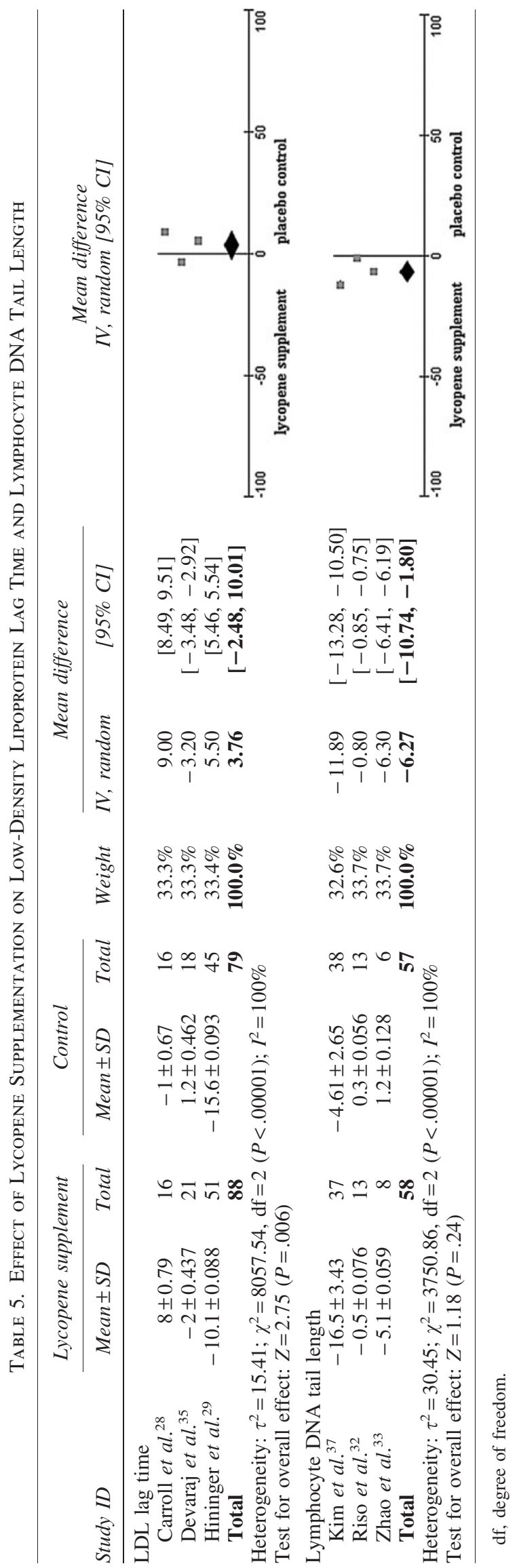

the quantitative meta-analysis for DNA tail length, the positive effect observed still offers an insight into the in vivo mechanism of action of lycopene and provides perspectives for consumers and researchers.

Mackinnon et $a l .{ }^{26}$ reported increased antioxidative capacity with lycopene supplementation and decreased lipid and protein oxidation products. Devaraj et al. ${ }^{35}$ reported decreased urinary DNA damage products, whereas Kim et $a l .{ }^{37}$ reported increased plasma SOD activity. No other significant effect was reported in these studies. Several factors contributed to the conflicting results of antioxidative activity studies. The null results might have resulted from the very low basal oxidative damage among the young healthy subjects who participated in several studies. ${ }^{27,29,31,32,37}$ Thus, basal variations are likely negligible and have little biological significance. Subjects with low baseline lycopene levels, such as those in the trial of Mackinnon et al. ${ }^{26}$ where the baseline lycopene level was $0.264 \mu \mathrm{M}$, may respond better than the subjects with elevated baseline lycopene levels. The dietary advice of the trials by Mackinnon et al. ${ }^{26}$ and Devaraj et al. ${ }^{35}$ both included refraining from lycopene-containing food for both the washout and the treatment period. The former instructed all participants to refrain from any vitamins and antioxidant supplementation, which could overestimate the magnitude of the effect.

Epidemiologic evidence and dietary intervention studies have associated tomato and lycopene with lower incidence rates of cardiovascular diseases and cancers. ${ }^{3,4,8}$ However, the effect of lycopene supplementation remains unconfirmed. Tomatoes contain significant amounts of $\beta$-carotene, vitamin $\mathrm{C}$, folate, and potassium. ${ }^{80}$ Thus, studies on the health benefits of tomatoes should consider other bioactive compounds that could contribute to the beneficial effects investigated.

\section{Oxidative stress and clinical outcomes}

Oxidative stress is often associated with high levels of reactive oxygen and oxidation products, particularly hydroperoxides and DNA fragments. ${ }^{1}$ Pinchuk et al. ${ }^{81}$ proposed several problems in the commonly used assays for ranking antioxidants, including that (1) the assays usually evaluate the effects of antioxidants that quench free radicals, which constitute only a part of the antioxidative network, and (2) antioxidative capacity and the potency of antioxidants, as determined by various methods, do not necessarily correlate with each other. Pinchuk et al. ${ }^{81}$ and Dotan et al. ${ }^{82}$ further suggested that the term oxidative stress of any given type is context dependent, and that the evaluation should be based on the use of the most sensitive probe or combination for oxidative damage. As shown in Table 1, we included five trials that measured DNA damage as the only biomarker, ${ }^{27,30-33}$ whereas one trial measured DNA damage, lipid and protein oxidation, and TAC. ${ }^{26}$ The contrasting findings on the particular oxidative stress parameters in chronic diseases highlight the importance of validating and standardizing biomarkers for oxidative stress. 
Among our included studies, Kucuk et al. ${ }^{30}$ recruited men with newly diagnosed prostate cancer. After intervention, the subjects in the lycopene intervention group had smaller tumors, less involvement of surgical margins and/or extraprostatic tissues with cancer, less diffuse involvement of the prostate by high-grade prostatic intraepithelial neoplasia, and lower PSA levels compared with subjects in the control group. However, no significant difference was found in DNA oxidation products, which implied that DNA oxidation might not be as critical and indicative in the progression of prostate cancer as it is in the occurrence of prostate cancer. Lycopene may also exert its anticarcinogenic effect via another pathway, such as by alteration of cell gap communication, as the authors suggested. Talvas et al. ${ }^{36}$ found that the PSA levels in healthy men were unchanged after lycopene intervention. This result also suggests the fact that the efficacy of lycopene might manifest more in the pathologic state. Kim et al. ${ }^{37}$ found that the beneficial effects of lycopene supplementation on endothelial function (i.e., RH-PAT, hs-CRP, and sVCAM-1) were remarkable among subjects with relatively impaired endothelial cell function. To some extent, this finding confirms the assumption that lycopene is more effective in the oxidative impaired population. However, the small number of qualified samples limits the correlation of oxidative stress and outcomes of cancer and cardiovascular diseases in this meta-analysis.

It is well documented that subjects with risk factors such as hypertension, diabetes, and smoking have increased levels of oxidative stress based on the rate of oxidant production and by elevated plasma markers for molecular oxidation such as lipid hydroperoxides. ${ }^{19,20}$ However, these oxidation products or biomarkers have not yet been adequately validated as markers for the onset, progression, and regression of any chronic diseases, which presents a challenge to the interpretation and integration of current evidence and justifies the ongoing research on oxidative biomarkers and antioxidant efficacies. ${ }^{12,83}$

\section{Strengths and limitations of this review}

The present systematic review has several advantages. This is the first systematic review that examines the effects of lycopene supplementation on oxidative stress in vivo. Also, a detailed research protocol was included, with prior specification and evaluation of potential study design considerations without language exclusion. The strengths of the study also include the analysis of only randomized, controlled clinical trials and the objective assessment of trial quality. Furthermore, variable biomarkers for oxidative stress were categorized for analysis.

Except for the uncertainty in evaluating oxidative stress biomarkers as discussed above, the following limitations warrant further discussion. First, the RCTs of lycopene supplementation with small quantities of other micronutrients were included, and not all lycopene supplements are equivalent. However, the same is true for all the manufactured lycopene supplements, whether purified or formulations. Thus, this review would still offer insights into the consumption of lycopene supplements. Second, this review includes one crossover trial; while in the presence of carryover, a common strategy is to base the analysis on the first period. The corresponding data were not a source of bias, but it usually decreases the weight of trials in metaanalyses. ${ }^{25}$ We also did not perform subgroup analysis because of the limited number of studies. Therefore, we were unable to confirm whether lycopene has different antioxidative effects among subjects with variable physiologic states, or whether lycopene may act differently in short-term versus long-term application, which remains an important area for future research.

\section{Conclusion and implication for practice and research}

The included trials used variable methods to measure oxidative stress, which caused incomparability across studies. Understanding the effectiveness of antioxidants in vivo requires identifying the markers or combinations of markers that are predictive of human diseases. Thus, biomarker research needs to move in parallel with lycopene intervention trials for disease prevention. In retrospect, dietary interventions using antioxidant supplements, lycopene in this case, may have been premature because of the need for more fundamental information on the mechanisms of action of these compounds. Given the current evidence, the supposition that lycopene is efficient as an antioxidant in vivo needs further examination. With the elucidation of the oxidative stress pathways, understanding the efficacy of lycopene could be clarified. Therefore, until further research establishes significant health benefits for lycopene supplementation in humans, it should be concluded that the consumption of natural carotenoid-rich fruits and vegetables is preferential to purified lycopene supplementation.

\section{ACKNOWLEDGMENTS}

This work was supported by the National Natural Science Foundation of China [Grant no. 81030053] and the National High-technology Research and Development Program (863 program) of China [Grant no. 2010AA023001].

\section{AUTHOR DISCLOSURE STATEMENT}

The authors declare that there is no conflict of interest.

\section{REFERENCES}

1. Sies H: Oxidative stress. Introductory remarks. In: Oxidative Stress (Sies, H, ed.), Academic Press, London, 1985, pp. 1-8.

2. Basu A, Imrhan V: Tomatoes versus lycopene in oxidative stress and carcinogenesis: conclusions from clinical trials. Eur J Clin Nutr 2007;61:295-303.

3. Cooke MS, Olinski R, Evans MD: Does measurement of oxidative damage to DNA have clinical significance? Clin Chim Acta 2006;365:30-49.

4. Willcox JK, Catignani GL, Lazarus S: Tomatoes and cardiovascular health. Crit Rev Food Sci 2003;43:1-18. 
5. Di Mascio P, Kaiser S, Sies H: Lycopene as the most efficient biological carotenoid singlet oxygen quencher. Arch Biochem Biophys 1989;274:532-538.

6. Stahl W, Sies H: Perspectives in biochemistry and biophysics, lycopene: a biologically important carotenoid in humans? Arch Biochem Biophys 1996;336:1-9.

7. Mayne ST: Beta-carotene, carotenoids, and disease prevention in humans. FASEB J 1996;10:690-701.

8. Gerster H: The potential role of lycopene for human health. $J$ Am Coll Nutr 1997;16:109-126.

9. Rao AV, Agarwal S: Role of antioxidant lycopene in cancer and heart disease. J Am Coll Nutr 2000;19:563-569.

10. Sies H, Stahl W, Sundquist AR: Antioxidant functions of vitamins: vitamin $\mathrm{E}$ and $\mathrm{C}$, b-carotene and other carotenoids. Ann NY Acad Sci 1992;669:7-20.

11. Arab L, Steck S: Lycopene and cardiovascular disease. Am J Clin Nutr 2000;71:1691S-1695S.

12. Johnson LJ, Meacham SL, Kruskall LJ: The antioxidantsvitamin $\mathrm{C}$, vitamin $\mathrm{E}$, selenium, and carotenoids. J Agromedicine 2003;9:65-82.

13. Erdman JW Jr, Ford NA, Lindshield BL: Are the health attributes of lycopene related to its antioxidant function? Arch Biochem Biophys 2009;483:229-235.

14. Paetau I, Chen H, Goh NM, White WS: Interactions in the postprandial appearance of $\beta$-carotene and canthaxanthin in plasma triacylglycerolrich lipoproteins in humans. Am J Clin Nutr 1997;66:1133-1143.

15. Kostic D, White WS, Olson JA: Intestinal absorption, serum clearance, and interactions between lutein and $\beta$-carotene when administered to human adults in separate or combined oral doses. Am J Clin Nutr 1995;62:604-610.

16. White WS, Stacewicz-Sapuntzakis M, Erdman JW Jr, Bowen PE: Pharmacokinetics of beta-carotene and canthaxanthin after ingestion of individual and combined doses by human subjects. $J$ Am Coll Nutr 1994;13:665-671.

17. Astley SB, Elliott RM, Archer DB, Southon S: Evidence that dietary supplementation with carotenoids and carotenoid-rich foods modulates the DNA damage: repair balance in human lymphocytes. Br J Nutr 2004;91:63-72.

18. Kavanaugh CJ, Trumbo PR, Ellwood KC: The U.S. Food and Drug Administration's evidence-based review for qualified health claims: tomatoes, lycopene, and cancer. J Natl Cancer Inst 2007;99:1074-1085.

19. Pandey KB, Rizvi SI: Markers of oxidative stress in erythrocytes and plasma during aging in humans. Oxid Med Cell Longev 2010;3:2-12.

20. Collins AR: Assays for oxidative stress and antioxidant status: applications to research into the biological effectiveness of polyphenols. Am J Clin Nutr 2005;81:261S-267S.

21. Higgins JPT, Green S (eds.): Cochrane Handbook for Systematic Reviews of Interventions. Wiley-Blackwell, Hoboken, NJ, 2008.

22. Schulz KF, Chalmers L, Hayes RJ, Altman DG: Empirical evidence of bias: Dimensions of methodological quality associated with estimates of treatment effects in controlled trials. JAMA 1995;273:408-412.

23. Follman D, Elliot P, Suh I, Cutler J: Variance imputation for reviews of clinical trials with continuous response. J Clin Epidemiol 1992;45:769-773.

24. Deeks JJ, Altman DG, Bradburn MJ: Statistical methods for examining heterogeneity and combining results from several studies in meta-analysis. In: Systematic Reviews in Health Care: MetaAnalysis in Context. 2nd ed. (Egger M, Davey Smith D, Altman DG, eds.). BMJ Publishing Group, London, 2001, pp. 289-301.

25. The Cochrane Collaboration. Review Manager (RevMan). Copenhagen: The Nordic Cochrane Centre, 2008.

26. Mackinnon ES, Rao AV, Josse RG, Rao LG: Supplementation with the antioxidant lycopene significantly decreases oxidative stress parameters and the bone resorption marker $\mathrm{N}$-telopeptide of type I collagen in postmenopausal women. Osteoporos Int 2011;22:1091-1101.

27. Collins AR, Olmedilla B, Southon S, Granado F, Duthie SJ: Serum carotenoids and oxidative DNA damage in human lymphocytes. Carcinogenesis 1998;19:2159-2162.

28. Carroll YL, Corridan BM, Morrissey PA: Lipoprotein carotenoid profiles and the susceptibility of low density lipoprotein to oxidative modification in healthy elderly volunteers. Eur J Clin Nutr 2000;54:500-507.

29. Hininger IA, Meyer-Wenger A, Moser U, et al: No significant effects of lutein, lycopene or b-carotene supplementation on biological markers of oxidative stress and LDL oxidizability in healthy adult subjects. J Am Coll Nutr 2001;20: 232-238.

30. Kucuk O, Sarkar FH, Djuric Z, et al.: Effects of lycopene supplementation in patients with localized prostate cancer. Exp Biol Med 2002;227:881-885.

31. Briviba K, Kulling SE, Möseneder J, Watzl B, Rechkemmer G, Bub A: Effects of supplementing a low-carotenoid diet with a tomato extract for 2 weeks on endogenous levels of DNA single strand breaks and immune functions in healthy non-smokers and smokers. Carcinogenesis 2004;25:2373-2379.

32. Riso P, Visioli F, Grande S, et al.: Effect of a tomato-based drink on markers of inflammation, immunomodulation, and oxidative stress. J Agric Food Chem 2006;54:563-566.

33. Zhao X, Aldini G, Johnson EJ, et al:: Modification of lymphocyte DNA damage by carotenoid supplementation in postmenopausal women. Am J Clin Nutr 2006;83:163-169.

34. Neyestani TR, Shariatzadeh N, Gharavi A, Kalayi A, Khalaji $\mathrm{N}$ : Physiological dose of lycopene suppressed oxidative stress and enhanced serum levels of immunoglobulin $\mathrm{M}$ in patients with Type 2 diabetes mellitus: a possible role in the prevention of long-term complications. J Endocrinol Invest 2007;30:833838 .

35. Devaraj S, Mathur S, Basu A, et al.: A dose-response study on the effects of purified lycopene supplementation on Bbiomarkers of oxidative stress. J Am Coll Nutr 2008;27:267-273.

36. Talvas J, Caris-Veyrat C, Guy L, et al.: Differential effects of lycopene consumed in tomato paste and lycopene in the form of a purified extract on target genes of cancer prostatic cells. Am J Clin Nutr 2010;91:1716-1724.

37. Kim JY, Paika JK, Kim OY, et al.: Effects of lycopene supplementation on oxidative stress and markers of endothelial function in healthy men. Atherosclerosis 2011;215:189-195.

38. van Breemen RB, Sharifi R, Viana M, et al.: Antioxidant effects of lycopene in African American men with prostate cancer or benign prostate hyperplasia: a randomized, controlled trial. Cancer Prev Res (Phila) 2011;4:711-718.

39. Pool-Zobel BL, Bub A, Müller H, Wollowski I, Rechkemmer G: Consumption of vegetables reduces genetic damage in humans: first results of a human intervention trial with carotenoid-rich foods. Carcinogenesis 1997;18:1847-1850. 
40. Rao AV, Agarwal S: Bioavailability and in vivo antioxidant properties of lycopene from tomato products and their possible role in the prevention of cancer. Nutr Cancer 1998;31:199-203.

41. Agarwal S, Rao AV: Tomato lycopene and low density lipoprotein oxidation: a human dietary intervention study. Lipids 1998;33:981-984.

42. Wright AJ, Hughes DA, Bailey AL, Southon S: Beta-carotene and lycopene, but not lutein, supplementation changes the plasma fatty acid profile of healthy male non-smokers. J Lab Clin Med 1999; 134:592-598.

43. Torbergsen AC, Collins AR: Recovery of human lymphocytes from oxidative DNA damage; the apparent enhancement of DNA repair by carotenoids is probably simply an antioxidant effect. Eur J Nutr 2000;39:80-85.

44. Bub A, Watzl B, Abrahamse L, et al.: Moderate intervention with carotenoid-rich vegetable products reduces lipid peroxidation in men. J Nutr 2000;130;2200-2206.

45. Pellegrini N, Riso P, Porrini M: Tomato consumption does not affect the total antioxidant capacity of plasma. Nutrition 2000;16:268-271.

46. Porrini M, Riso P: Lymphocyte lycopene concentration and DNA protection from oxidative damage is increased in women after a short period of tomato consumption. J Nutr 2000;130:189-192.

47. Chen L, Stacewicz-Sapuntzakis M, Duncan C, et al.: Oxidative DNA damage in prostate cancer patients consuming tomato sauce-based entrees as a whole-food intervention. J Natl Cancer Inst 2001;93:1872-1879.

48. Rao AV, Shen H: Effect of low dose lycopene intake on lycopene bioavailability and oxidative stress. Nutr Res 2002;22:1125-1131.

49. Porrini M, Riso P, Oriani G: Spinach and tomato consumption increases lymphocyte DNA resistance to oxidative stress but this is not related to cell carotenoid concentrations. Eur J Nutr 2002;41:95-100.

50. Visioli F, Riso P, Grande S, Galli C, Porrini M: Protective activity of tomato products on in vivo markers of lipid oxidation. Eur J Nutr 2003;42:201-206.

51. Riso P, Visioli F, Erba D, Testolin G., Porrini M: Lycopene and vitamin $\mathrm{C}$ concentrations increase in plasma and lymphocytes after tomato intake. Effects on cellular antioxidant protection. Eur J Clin Nutr 2004;58:1350-1358.

52. Rao AV: Processed tomato products as a source of dietary lycopene: bioavailability and antioxidant properties. Can J Diet Pract Res 2004;65:161-165.

53. Madrid AE, Vásquez ZD, Leyton AF, Mandiola C, Escobar F JA: Short-term Lycopersicum esculentum consumption may increase plasma high density lipoproteins and decrease oxidative stress. Rev Méd Chile 2006;134:855-862.

54. Goyal A, Chopra Ma, Lwaleed BA, Birch B, Cooper AJ: The effects of dietary lycopene supplementation on human seminal plasma. BJU International 2007;99:1456-1460.

55. Bose KS, Agrawal BK: Effect of lycopene from tomatoes (cooked) on plasma antioxidant enzymes, lipid peroxidation rate and lipid profile in grade-I hypertension. Ann Nutr Metab 2007;51:477-481.

56. Rao LG, Mackinnon ES, Josse RG, Murray TM, Strauss A, Rao AV: Lycopene consumption decreases oxidative stress and bone resorption markers in postmenopausal women. Osteoporos Int 2007;18:109-115.

57. Silaste ML, Alfthan G, Aro A, Kesäniemi YA, Hörkkö S: Tomato juice decreases LDL cholesterol levels and increases LDL resistance to oxidation. Br J Nutr 2007;98:1251-1258.
58. Engelhard YN, Gazer B, Paran E, Sheva B: Natural antioxidants from tomato extract reduce blood pressure in patients with grade1 hypertension: A double-blind, placebo-controlled pilot study. Am Heart J 2006;151:100.e1-e6.

59. Denniss SG, Haffner TD, Kroetsch JT, Davidson SR, Rush JW, Hushson RL: Effect of short-term lycopene supplementation and postprandial dyslipidemia on plasma antioxidants and biomarkers of endothelial health in young, healthy individuals. Vasc Health Risk Manag 2008;4:213-222.

60. Martínez-Tomás R, Larqué E, González-Silvera D, SánchezCampillo M, Burgos MI, Wellner A: Effect of the consumption of a fruit and vegetable soup with high in vitro carotenoid bioaccessibility on serum carotenoid concentrations and markers of oxidative stress in young men. Eur J Nutr 2012;51:231-239.

61. Böhm V, Bitsch R: Intestinal absorption of lycopene from different matrices and interactions to other carotenoids, the lipid status, and the antioxidant capacity of human plasma. Eur J Nutr 1999;38:118-125.

62. Ahuja KD, Kunde D, Ball MJ: Effects of olive oil and tomato lycopene combination on heart disease risk factors. Asia Pac J Clin Nutr 2003;12 Suppl:S21-S26.

63. Collins JK, Arjmandi BH, Claypool PL, Perkins-Veazie1 P, Baker RA, Clevidence BA: Lycopene from two food sources does not affect antioxidant or cholesterol status of middle-aged adults. J Nutr 2004;3:15-20.

64. Ahuja KD, Pittaway JK, Ball MJ: Effects of olive oil and tomato lycopene combination on serum lycopene, lipid profile, and lipid oxidation. Nutrition 2006;22:259-265.

65. Shen YC, Chen SL, Wang CK: Contribution of tomato phenolics to antioxidation and down-regulation of blood lipids. J Agric Food Chem 2007;55:6475-6481.

66. Jacob K, Periago MJ, Bohm V, Berruezo GR: Influence of lycopene and vitamin $\mathrm{C}$ from tomato juice on biomarkers of oxidative stress and inflammation. Br J Nutr 2008;99:137-146.

67. Steinberg FM, Chait A: Antioxidant vitamin supplementation and lipid peroxidation in smokers. Am J Clin Nutr 1998;68:319-327.

68. Sutherland WH, Walker RJ, De Jong SA, Upritchard JE: Supplementation with tomato juice increases plasma lycopene but does not alter susceptibility to oxidation of low-density lipoproteins from renal transplant recipients. Clin Nephrol 1999;52:30-36.

69. Riso P, Pinder A, Santangelo A, Porrini M: Does tomato consumption effectively increase the resistance of lymphocyte DNA to oxidative damage? Am J Clin Nutr 1999;69:712-718.

70. Chopra M, O'Neill ME, Keogh N, Wortley G, Southon S, Thurnham DI: Influence of increased fruit and vegetable intake on plasma and lipoprotein carotenoids and LDL oxidation in smokers and nonsmokers. Clin Chem 2000;46:1818-1829.

71. Upritchard JE, Sutherland WH, Mann JI: Effect of supplementation with tomato juice, vitamin E, and vitamin C on LDL oxidation and products of inflammatory activity in type 2 diabetes. Diabetes Care 2000;23:733-738.

72. Arab L, Steck-Scott S, Fleishauer AT: Lycopene and the lung. Exp Biol Med 2002;227:894-899.

73. Bub A, Barth S, Watzl B, et al.: Paraoxonase 1 Q192R (PON1192) polymorphism is associated with reduced lipid peroxidation in R-allele-carrier but not in QQ homozygous elderly subjects on a tomato-rich diet. Eur J Nutr 2002;41:237-243.

74. Hadley CW, Clinton SK, Schwartz SJ: The consumption of processed tomato products enhances plasma lycopene concen- 
trations in association with a reduced lipoprotein sensitivity to oxidative damage. J Nutr 2003;133:727-732.

75. Briviba K, Schnäbele K, Rechkemmer G, Bub A: Supplementation of a diet low in carotenoids with tomato or carrot juice does not affect lipid peroxidation in plasma and feces of healthy men. J Nutr 2004;134:1081-1083.

76. Bub A, Barth SW, Watzl B, Briviba K, Rechkemmer G: Paraoxonase 1 Q192R (PON1-192) polymorphism is associated with reduced lipid peroxidation in healthy young men on a low carotenoid diet supplemented with tomato juice. Br J Nutr 2005;93:291-297.

77. Paterson E, Gordon MH, Niwat C, et al.: Supplementation with fruit and vegetable soups and beverages increases plasma carotenoid concentrations but does not alter markers of oxidative stress or cardiovascular risk factors. J Nutr 2006;136:2849-2855.

78. Linseisen J, Hoffmann J, Riedl J, Wolfram G: Effect of a single oral dose of antioxidant mixture (vitamin E, carotenoids) on the formation of cholesterol oxidation products after ex vivo LDL oxidation in humans. Eur J Med Res 1998;21:5-12.

79. Hoffmann J, Linseisen J, Riedl J, Wolfram G: Dietary fiber reduces the antioxidative effect of a carotenoid and a-tocopherol mixture on LDL oxidation ex vivo in humans. Eur J Nutr 1999;38:278-285.

80. Beecher GR: Nutrient content of tomatoes and tomato products. Proc Soc Exp Biol Med 1998;218:98-100.

81. Pinchuk I, Shoval H, Dotan Y, Lichtenberg D: Evaluation of antioxidants: scope, limitations and relevance of assays. Chem Phys Lipid 2012;165:638-647.

82. Dotan Y, Lichtenberg D, Pinchuk I: Lipid peroxidation cannot be used as a universal criterion of oxidative stress. Prog Lipid Res 2004;43:200-227.

83. Bruckdorfer KR: Antioxidants and CVD. Proc Nutr Soc 2008;67:214-222. 